\title{
Urgences
}

\section{Paul-Marie Lapointe : " La mission du croisé "}

\section{André Marquis}

Numéro 23, avril 1989

Lisière du livre

URI : https://id.erudit.org/iderudit/025518ar

DOI : https://doi.org/10.7202/025518ar

Aller au sommaire du numéro

Éditeur(s)

Urgences

ISSN

0226-9554 (imprimé)

1927-3924 (numérique)

Découvrir la revue

Citer cet article

Marquis, A. (1989). Paul-Marie Lapointe : " La mission du croisé ". Urgences, (23), 70-76. https://doi.org/10.7202/025518ar d'utilisation que vous pouvez consulter en ligne.

https://apropos.erudit.org/fr/usagers/politique-dutilisation/ 
Mots croisés et case vide

écRiturEs de Paul-Marie Lapointe

Dieu de Carole Massé

Pour une esthétique de la pédagogie de Jacques Daignault 


\section{ANDRÉ MARQUIS \\ Paul-Marie Lapointe: "La mission du croisé»}

[... ] une simple mention comme Premier volume ou Tome 1 a force de promesse - ou, comme dit Northrop Frye, de «menace".

\section{Gérard Genette}

L'entreprise la plus singulière de Lapointe est sûrement écRiturEs, publié en 1980 (l'année des désillusions indépendantistes!), qui se moque des règles élémentaires de la communication et de la poétique. Lapointe affirme avoir composé ces textes à partir des mots croisés que l'on trouve dans les journaux ou que l'on vend en recueil à la tabagie du coin. À première vue, les poèmes présentent les caractéristiques du code: définitions, réponses, synonymes, abréviations, lieux historiques, personnages célèbres. De plus, l'inventaire semble limité, puisque les mêmes définitions reviennent d'un poème à l'autre, comme cela arrive fréquemment dans un recueil de mots croisés. Les réseaux isotopes se font rares. Les mots n'ont d'autre motivation que leur coexistence sur la page; ils s'unissent dans cette seule relation de contiguïté. Lapointe cherche à tuer le sens; il aligne des mots pour l'unique plaisir d'écrire, d'où le titre de son recueil'.

écRiturEs se présente dans un coffret solide et attrayant. Sur un fond de couleur gris-bleu, sont tracés de façon très stylisée et déployés dans toutes les directions, différents idéogrammes (?) rouges, bleu royal ou jaunes. On reconnaît le $c$, le $f$, le $p$, le $s$, le $t$ et le $\boldsymbol{u}$; on devine un $a$, un $e$, un $\boldsymbol{i}$, un $\boldsymbol{m}$, un $n$, ainsi que quelques signes de ponctuation comme l'apostrophe, le guillemet, le point, le point d'exclamation et la virgule. On peut reconstituer presque intégralement le nom de l'auteur, Paul-Ma[r]ie Lapointe. Certains y verront des lettres grecques, comme le delta, l'epsilon et le mu, et une note musicale. D'autres lettres, d'alphabets que je ne connais pas, y sont peut-être représentées. Ces «dessins» aux couleurs vives créent une impression de mouvement et de légèreté. Ils tranchent nettement avec la sobriété noire, grise et blanche des lettres disposées de façon conventionnelle et statique au dos des deux livres. Outre l'effet commercial recherché (l'acheteur potentiel est obligé de se procurer les deux tomes à la fois ${ }^{2}$ ), le coffret ajoute une valeur symbolique aux textes puisqu'on a pris la peine de les «recouvrir». Habituellement, seuls les livres faisant partie d'une collection luxueuse se vendent en coffret, comme ceux de la 
prestigieuse «Bibliothèque de la Pléiade». On ne peut pas dire que la qualité de la présentation matérielle d'écRiturEs (papier ordinaire, texte dactylographié, etc.) justifie l'usage du coffret. Je crois qu'il faut y voir, par-delà l'effet pratique, un acte ironique compte tenu du type d'écriture expérimenté par Lapointe.

Jetons maintenant un coup d'oeil au titre. Le mot écRiturEs, séparé après le $\mathbf{i}$, est réparti sur deux lignes, sans trait d'union (comme s'il s'agissait de deux mots différents). Sur le tome 1, la première partie, écRi, apparaît en noir, tandis que turEs est de couleur grise (selon le modèle de couleur employé dans les mots croisés?); puis ces couleurs s'inversent sur le tome 2 (la première partie du mot est grise, et la seconde, noire). La raison de cette présentation est très simple. Chaque lettre du mot écRiturEs sert à titrer un "cahier ${ }^{3}$ de l'ouvrage qui en contient 9 . Le premier tome regroupe les quatre premières lettres, le second tome, les cinq dernières. La couleur noire indique donc les lettres du mot écRiturEs qui figurent à l'intérieur de chaque tome. Comme il n'y a pas de piste isotopique forte d'un cahier à l'autre, d'un texte à l'autre, d'un mot à l'autre, l'auteur n'a pas senti la nécessité d'unir les deux composantes du mot par le trait d'union conventionnel. Chaque élément est autonome et se suffit à lui-même. Ce titre rhématique ${ }^{4}$ n'a rien d'accrocheur, seule l'utilisation inhabituelle des majuscules lui donne un cachet énigmatique ${ }^{5}$. Alors que les écrivains disent faire de la poésie, du roman, de la fiction, du texte, de l'essai, Paul-Marie Lapointe, lui, fait de l'écriture. Voilà un nouveau terme dans la guerre des genres!

Les troisième et huitième lettres du titre (les avant-dernières de chaque tome) sont reproduites en majuscule et forment la syllabe «RE». Faut-il y voir l'ablatif du mot latin «res» qui signifie «au sujet de» ou "ce qui est en question?" ${ }^{6}$. S'agit-il du préfixe signifiant "à nouveau» et qui nous inciterait à lire écritures et récritures? Si les lettres «RE» apparaissent dans le prénom de l'auteur, Marie, on ne retrouve pas en majuscules les autres lettres constitutives de son nom. Cette piste ne mène donc nulle part. Dans la grille des mots croisés, «RE» forme un mot passe-partout qui répond aux questions de quatre catégories différentes $\left.{ }^{7}: 1\right)$ préfixe; 2) note, note de musique ou dans la gamme; 3 ) île (française) de l''Atlantique ou île voisine d'Oléron et 4) dieu solaire ou dieu des Égyptiens. II faut retenir ce pluri-sémantisme qui est à la base du jeu de lettres. L'auteur tente d'éloigner le mot de son sens courant pour l'intégrer de façon surprenante à un poème. L'étonnement est garanti. 
Une autre conséquence de la disposition est qu'on peut lire certains mots à la verticale (et $\left.{ }^{8}, \mathrm{cu}(\mathrm{I}), \mathrm{re}\right)^{9}$. La liste s'allonge considérablement si on se laisse aller à lire dans tous les sens, en ne tenant compte que de la contiguïté des lettres (écurie[s], écu, écrire, eut, curie[s], cet, cru[e], crut, cri[s], crie[s], cré, cure[s], rut, rue, ri, rie[s], rire[s], ire[s], sire, écru, crise, té, tec, tu, tué, etc.). Je ne parviens pas à trouver une signification par ailleurs plus importante aux textes réunis dans les deux parties désignées par une lettre majuscule. De même, j'ignore la raison qui détermine l'inclusion de tel poème dans une partie du recueil plutôt que dans une autre. Est-ce moi qui suis aveugle?

Le nom de la maison d'édition, l'Obsidienne, signifie: «Nom de diverses variétés de laves (rhyolite, trachyte et andésite) ressemblant au verre, de couleur foncée. ${ }^{10}$. Dans écRiturEs, les mots coulent comme la lave, comme la matière en fusion. Ce nom recherché, technique, démontre l'érudition de la personne qui l'a choisi ${ }^{11}$. On ne s'étonnera pas de remarquer que le nom de l'auteur est écrit en plus gros caractères que les autres indications au dos du livre. Le titre est banal, le nom de la maison d'éditien, peu connu $^{12}$. La crédibilité de l'entreprise repose sur les épaules de l'auteur, personnage littéraire célèbre et fonctionnaire haut placé à Radio-Canada ${ }^{13}$. En mettant l'accent sur les «procédés d'écriture» et les "exercices d'écriture» devenus, justement par déplacement du s, le titre - écRiturEs - , Paul-Marie Lapointe désacralise la notion d'écriture, faisant ainsi un pied de nez aux écritures bibliques $^{14}$ et aux écritures comptables ${ }^{15}$. Entreprise de démystification ou fumisterie?

La première de couverture est d'une sobriété exemplaire: le nom de l'auteur, le titre, l'indication du tome et, au bas, le nom de la maison d'édition, tous justifiés par rapport à la marge de gauche. Les deux majuscules du titre surprennent le lecteur et retiennent son attention. Sur la quatrième de couverture, on retrouve des extraits d'un texte que Lapointe a publié dans la Nouvelle barre du jour en 1977. Double caution. Celle de l'auteur encore, qui explique ce qu'il a voulu faire, et celle d'un lieu d'édition d'avant-garde à cette époque. Ce texte, qui fait office de préface, indique au lecteur de quelle façon il doit aborder écRiturEs. Sur le tome 1, Lapointe insiste sur la fabrication de l'ouvrage, conçu à partir des mots croisés. En laissant totale liberté aux mots, il croit parvenir à déjouer le sens. Curieusement, sur le tome 2, Lapointe termine son texte en écrivant que «la liberté des mots préfigure la liberté des hommes". Ce thème n'est-il pas au coeur de tous les recueils de Lapointe? L'année de publication de ces extraits nous indique que 
le projet de Lapointe remonte au moins à 1977. II s'est donc écoulé trois ans avant la publication de l'ouvrage, ce qui pourrait être un signe, sinon de réussite, du moins du sérieux de l'entreprise. Le nom de l'auteur apparaît donc sur les trois composantes de la couverture.

Si Lapointe n'hésite pas à inscrire son nom sur les deux tomes (il revient à neuf reprises en considérant la couverture, la page de titre et la description signalétique), il se refuse à présenter la liste de ses recueils antérieurs. Cette omission n'est pas gratuite, Lapointe désirant sans doute que le lecteur lise écRiturEs sans idées préconçues, qu'il ne considère pas ce recueil comme le prolongement de ses livres précédents. Mais, à moins d'employer un pseudonyme, Lapointe ne sera jamais lu de cette façon idéale. Chacun de ses livres marque une étape dans son cheminement intellectuel et institutionnel. Mais n'est-ce pas ce que le public attend de Paul-Marie Lapointe, qu'il se renouvelle sans cesse?

Nous l'avons dit, les lettres du mot écRiturEs constituent les titres des différents cahiers du recueil. Les huit premiers cahiers regroupent chacun 100 poèmes (Le vierge incendié contient lui aussi 100 poèmes!), alors que le dernier n'en compte que 89 (dont 53 sont des variantes visuelles de trois poèmes), soit un grand total de 889 poèmes $^{16}$. Pourquoi Lapointe a-t-il rompu ainsi sa structure? On dirait que la machine textuelle s'est enrayée avant terme. Huit, huit, neuf, est-ce que ces chiffres indiquent la fin de la progression? La neuvième partie se termine donc sur le chiffre neuf.

Pourquoi Lapointe a-t-il tenu à ce que l'impression de ses textes reproduise le manuscrit dactylographié? Luc Bouvier mentionne que ce «procédé donne une grande liberté à l'auteur puisqu'il acquiert ainsi la capacité de disposer le texte à sa fantaisie, d'en changer aussi souvent qu'il le désire, le caractère d'imprimerie et l'intensité de l'encre, en somme d'en varier à son gré la présentation visuelle» ${ }^{17}$. Robert Melançon insiste sur le fait que ce type d'impression «rend sensible la matérialité de l'inscription» et confère à certaines pages «une plasticité plus riche ${ }^{18}$. J'ajouterai que la dactylographie rend plus "réel» l'effet de «mots croisés" et qu'elle véhicule à son tour l'idée de travail scolaire que comportent les mots "cahiers» et «écRiturEs". Pour Lapointe, seule importe l'écriture brute. La typographie aurait nui au lecteur dans sa relation rugueuse au poème ${ }^{19}$.

À la lecture d'écRiturEs, j'ai constaté quelques variantes d'une partie à l'autre. Ainsi, le cahier "c» exploite davantage le 
poème en prose et s'apparente en cela au Vierge incendié. Il en découle la même impression d'étrangeté et de non-sens. Parce que des liens syntaxiques relient les diverses propositions de la phrase, le lecteur perd de vue le jeu des questions-réponses. Le rythme est moins saccadé que dans la première partie. Lapointe tente une opération de camouflage et nous lance sur de multiples pistes de lecture. L'influence des mots croisés se fait moins sentir parce que l'auteur a décidé de recourir à une syntaxe plus orthodoxe et à des effets poétiques reconnus. Parmi ces procédés, l'allitération occupe une des premières places: "l'éclat de rire au reins / insère dans les raies de la reine» (poème 160).

Lapointe tente aussi des expériences de déconstruction spatiale. II défie le concept de vers en disposant certains textes de façon très originale sur la page. On notera des acrostiches (poème 51 ), des blancs séparant chaque lettre ${ }^{20}$ (poème 23), des calligrammes non figuratifs aux formes plus ou moins éclatées (poème 84), des lectures pluri-directionnelles ${ }^{21}$ (poème 56), des présentations cruciverbistes (poème 71). Bref, Lapointe prend toutes les libertés, se permet tous les écarts. Plusieurs poèmes d'écRiturEs s'inscrivent dans le courant de la poésie concrète.

Lapointe critique son travail et multiplie les mises en garde au lecteur: "Attention, si cela change, il s'agit certainement d'un truc. (...] S'agit-il d'un effort littéraire ou d'une possibilité de pouvoir?" (poème 181). Je répondrai que l'effort littéraire d'écRiturEs consiste à revaloriser le langage qui subit quotidiennement les affres de la communication claire et rapide. Lapointe démontre ainsi l'aspect du principe de pertinence des échanges linguistiques. II agence les mots pour notre plus grande surprise. Pourtant ce travail poétique ne pouvait être le fruit d'un auteur dépourvu d'un fort capital symbolique. Lapointe est reconnu depuis longtemps comme l'un des poètes majeurs de notre littérature. En ce sens il peut se permettre la publication d'un tel livre, sans se couvrir de ridicule. Il a même obtenu une réception étonnante compte tenu de la difficulté de lecture de son recueil. Quel jeune poète aurait pu investir (dans les deux sens du terme) dans un projet d'une telle envergure ${ }^{21}$ ? Lapointe n'ironise pas lorsqu'il écrit: «mon travail a saveur rude et désagréable / pénible» (poème 161).

Lire ces deux tomes sans pause relève de l'aliénation. L'émotion n'y circule pas à fleur de mots. Froide, lente, distante, voilà qui conviendrait mieux pour dépeindre cette écriture. Le lecteur se lasse rapidement des effets-chocs, qui perdent leur portée et se banalisent par leur trop grande récurrence. On ne sait pas quand 
l'écriture s'arrêtera, elle paraît intarissable. II faut plonger dans ce texte en acceptant les règles du jeu et en prenant le risque de ne pas tout comprendre. Le lecteur a droit à une expérience poétique à la limite du recevable. Priment ici la prouesse littéraire et le plaisir du jeu.

1. Paul-Marie Lapointe disait en interview à Robert Melançon: «ÉcRiturEs a été fait de soir en soir, systématiquement, simplement, pendant des mois... Écrire. D'où le titre. Ne pas vouloir dire!» "L'injustifiable poésie», Études françaises, Montéral, vol. 16, no 2, avril 1980, p. 98.

2. II n'aurait peut-être pas acheté le second après avoir feuilleté le premier!

3. Dans la description signalétique, Lapointe emploie le mot "cahier» pour désigner les différentes parties de son «ouvrage» (constitué de deux tomes). "Cahier" connote l'idée de travail scolaire, d'exercice régulier et assidu. Le terme "cahier» est aussi en relation directe avec le titre qui, loin de faire poétique, ramène l'écriture à sa dimension technique, à sa matérialité immédiate.

4. Genette distingue le titre thématique (subjectif) et le titre rhématique (objectif) de la façon suivante: le premier indique ce dont on parle (l'amour, la mort, etc.), tandis que le second indique de quoi il s'agit (poésie, théâtre, etc.). Seuils, coll. "Poétique», Paris, Seuil, 1987, p. 75, 76 et 85.

5. Il est cependant assez exceptionnel que la somme des intertitres équivaille au titre de l'ouvrage.

6. "Re» désigne aussi une "chose matérielle", un "objet naturel», un "fait historique"; ; bref, ce petit mot représente une foule de choses! J'ai consulté à cet effet Henri Goelzer, Dictionnaire latin-français, coll. "Le latin en poche», Paris, Garnier, 1928, p. 566.

7. Le Petit Robert 1 précise que «RE» peut aussi représenter l'abréviation du mot rhénium. Mais on ne retrouve pas ce mot dans le recueil de Lapointe, ni dans les mots croisés que j'ai effectués.

8. Cette conjonction de coordination remplacerait-elle le trait d'union absent?

9. Le second $\boldsymbol{r}$ du titre se trouve écrasé par le premier en majuscule.

10. Le Petit Robert 1, p. 1295.

11. Le lecteur se souviendra peut-être du célèbre vers de Roland Giguère («La poésie est une lampe d'obsidienne») écrit en 1965 et ainsi interprété par Paul Chamberland ("Lampe d'obsidienne", La barre du jour, Montréal, no 11-13 (no intitulé Connaissance de Giguère), décembre 1967-mai 1968, p. 43): «La "lampe d'obsidienne" réalise la "concrétion" de la lumière noire, le couple violent Midi/Minuit, la résolution de la contradiction nocturne/diurne». On retrouve ce vers dans la troisième rétrospective de Giguère: Forêt vierge folle, coll. "Parcours", Montréal, l'Hexagone, 1978, p. 112.

12. Ce livre (en 2 tomes) est le troisième publié à cette enseigne, après Bouche rouge (1976) et Tombeau de René Crevel (1979).

13. L'engouement médiatique pour écRiturEs n'est donc pas surprenant. Si les critiques ont insisté sur l'importance sociologique de cette publication, peu de personnes ont abordé le texte.

14. On dit alors Les Écritures, avec un grand é.

15. On emploie alors l'expression «tenir les écritures».

16. De nombreuses erreurs se sont glissées dans la table des matières en ce qui a trait à la distribution des majuscules et de certains titres. La plus importante apparaît dans la section titrée «Dactylologie» (étrangement, ce titre n'est pas repris à l'intérieur de l'ouvrage) et concerne le nombre de poèmes. L'éditeur écrit que la série de calligrammes se termine à la page 888, alors qu'elle prend 
fin à la page 889. Y a-t-il un calligramme de trop dans cette section? Lapointe voulait-il terminer son ouvrage sur un nombre qui contient trois fois le signe de l'infini mathématique (présenté à la verticale plutôt qu'à l'horizontale)?

17. Luc Bouvier, "Paul-Marie Lapointe. Écritures", Livres et auteurs québécois 1980, Québec, PUL, 1981, p. 114.

18. Robert Melançon, Paul-Marie Lapointe, coll. «Poètes d'aujourd'hui», no 254, Paris, Seghers, 1987, p. 79.

19. Comme Lapointe s'est auto-édité, le coût de la typographie n'aurait fait qu'augmenter la somme déjà considérable qu'il a dû investir dans cette publication.

20. Ce qui a pour effet de perturber considérablement la lecture, puisque le lecteur doit fournir un effort supplémentaire pour reconstituer chaque mot.

21. Soit horizontales, verticales et diagonales.

22. Quel éditeur aurait publié ces deux briques? Lapointe aurait-il accepté de faire paraître une version abrégée d'écRiturEs? II a contourné le problème en se publiant lui-même, sans faire de concessions esthétiques ou idéologiques. 